\title{
Ultrasensitive electrospun fluorescent nanofibrous membrane for rapid visual colorimetric detection of $\mathrm{H}_{2} \mathrm{O}_{2}$
}

\author{
Anitha Senthamizhan ${ }^{1} \cdot$ Brabu Balusamy $^{1} \cdot$ Zeynep Aytac $^{1,2} \cdot$ Tamer Uyar $^{1,2}$
}

Received: 21 July 2015 /Revised: 30 September 2015 / Accepted: 23 October 2015 / Published online: 4 December 2015

(C) Springer-Verlag Berlin Heidelberg 2015

\begin{abstract}
We report herein a flexible fluorescent nanofibrous membrane (FNFM) prepared by decorating the gold nanocluster (AuNC) on electrospun polysulfone nanofibrous membrane for rapid visual colorimetric detection of $\mathrm{H}_{2} \mathrm{O}_{2}$. The provision of AuNC coupled to NFM has proven to be advantageous for facile and quick visualization of the obtained results, permitting instant, selective, and on-site detection. We strongly suggest that the fast response time is ascribed to the enhanced probabilities of interaction with AuNC located at the surface of NF. It has been observed that the color change from red to blue is dependent on the concentration, which is exclusively selective for hydrogen peroxide. The detection limit has been found to be $500 \mathrm{nM}$ using confocal laser scanning microscope (CLSM), visually recognizable with good accuracy and stability. A systematic comparison was performed between the sensing performance of FNFM and AuNC solution. The underlying sensing mechanism is
\end{abstract}

Published in the topical collection Fiber-based Platforms for Bioanalytics with guest editors Antje J. Baeumner and R. Kenneth Marcus.

Anitha Senthamizhan and Brabu Balusamy contributed equally to this work.

Electronic supplementary material The online version of this article (doi:10.1007/s00216-015-9149-5) contains supplementary material, which is available to authorized users.

Anitha Senthamizhan

senthamizhan@unam.bilkent.edu.tr

Tamer Uyar

tamer@unam.bilkent.edu.tr; uyar@unam.bilkent.edu.tr; tameruyar@gmail.com

1 UNAM-National Nanotechnology Research Center, Bilkent University, 06800 Ankara, Turkey

2 Institute of Materials Science and Nanotechnology, Bilkent University, 06800 Ankara, Turkey demonstrated using UV spectra, transmission electron microscopy (TEM), and X-ray photoelectron spectroscopy (XPS). The corresponding disappearance of the characteristic emissions of gold nanoclusters and the emergence of a localized surface plasmon resonance (LSPR) band, stressing this unique characteristic of gold nanoparticles. Hence, it is evident that the conversion of nanoparticles from nanoclusters has taken place in the presence of $\mathrm{H}_{2} \mathrm{O}_{2}$. Our work here has paved a new path for the detection of bioanalytes, highlighting the merits of rapid readout, sensitivity, and user-friendliness.

Keyword Electrospinning - Nanofiber - Gold nanocluster · Fluorescence $\cdot$ Hydrogen peroxide $\cdot$ Sensor

\section{Introduction}

Hydrogen peroxide $\left(\mathrm{H}_{2} \mathrm{O}_{2}\right)$ has vital roles in the regulation of many physiological processes and is widely used for numerous applications, including food processing, paper bleaching, antiseptic, and disinfecting agents [1-3]. However, overproduction of $\mathrm{H}_{2} \mathrm{O}_{2}$ causes several toxic pathologic events from aging to severe diseases, including cancer and neurodegenerative Alzheimer's, Parkinson's diseases [4]. Thus, recent years have attracted attention regarding the measurement of $\mathrm{H}_{2} \mathrm{O}_{2}$ for environmental protection and human health. Until the recent past, various analytical techniques have been devised for the analysis of $\mathrm{H}_{2} \mathrm{O}_{2}$, including chemiluminescence, spectrophotometry, fluorescence, and electrochemical methods [5-8]. Although these methods offer high sensitivity, rapidity, they also pose disadvantages like increased time consumption, increased operating procedures, expensive reagents, high cost, and the instability of substrates.

Recent studies have indicated the emergence of fluorescence-based methods, attracting great interest because 
of their sensitivity, selectivity, fast response, and ease of use [9-11]. However, there still exists a challenge of developing a simple, highly sensitive, and inexpensive platform for the rapid detection of $\mathrm{H}_{2} \mathrm{O}_{2}$ [12, 13]. In-depth analyses and studies have pointed out electrospun-based nanofibers to be superior to the conventional ones as sensing devices [14-17]. Owing to the flexible nature of nanofibrous membranes, these sensors have gained popularity to detect $\mathrm{H}_{2} \mathrm{O}_{2}$ using various approaches, which might also be applied in the practical field in the near future.

The fluorescent metal nanoclusters (FMNC) are extensively studied and successfully applied in sensing application because of their flexibilities in terms of modification of the surface with a variety of functional groups, including thiols, polymers, dendrimer, DNA, and peptides. The prominent sensitivity nature of the nanoclusters has been highlighted for the successful development of effective sensors, making them fitting as functional materials. Among the various nanoclusters, bovine serum albumin capped nanoclusters (AuNC) have been extensively used in the fields of sensing and bio-imaging because of their nontoxicity and good photostability [18-21]. Therefore, the design and further development of a FMNC-based colorimetric sensing strategy has gained sufficient popularity and is strongly encouraged. Recent times have seen the integration of FMNC onto a support for the development of sensors to overcome the limitations of solution-based sensors. Several nanomaterials, including silica, carbon nanotubes, etc., have been used as a template for holding FMNC.

Recent studies demonstrated electrospun nanofibers as an excellent matrix for protecting and enhancing the sensing performance of fluorescent gold nanoclusters and metallic nanoparticles [22-26]. The polysulfone fiber-based membranes received much attention in the field ranging from artificial kidneys for blood purification to water treatment and food processing, owing to their good permeability, mechanical, thermal, and biocompatible properties [27, 28]. Until now, most of the sensor probes have been designed based on a chemical reaction approach, which considers taking a specific reaction towards the targeted analytes. This marks the importance of proper optimization of fluorescent probe in understanding the sensing and selective performances. Mostly, the sensing of these analytes requires long reaction time to achieve signal output, leading to a potential influence on the real-time monitoring and quantification. This poses a serious challenge to design efficient probes that can rapidly respond to $\mathrm{H}_{2} \mathrm{O}_{2}$. However, the rapid response time of electrospun fibrous membrane-based colorimetric sensors have not yet been demonstrated, to the best of our knowledge. We speculated that the synergetic combinations of the sensing performances of AuNC and electrospun nanofibers would set the right path towards the rapid detection of $\mathrm{H}_{2} \mathrm{O}_{2}$.

In the present investigation, we have schematically described the electrospun fluorescent nanofiber-based visual colorimetric detection of $\mathrm{H}_{2} \mathrm{O}_{2}$. A quick color change has been observed from bright red to blue. It has been observed that the characteristic peak of AuNC disappears and a new peak emerges with an absorption maximum around $546 \mathrm{~nm}$, upon interaction with $\mathrm{H}_{2} \mathrm{O}_{2}$. This is due to its well-known optical property of gold nanoparticles, called "LSPR" band. The outcome suggests the formation of nanoparticles from gold nanoclusters.

\section{Materials and methods}

Tetrachloroauric acid trihydrate $\left(\mathrm{HAuCl}_{4} \cdot 3 \mathrm{H}_{2} \mathrm{O}\right)$, bovine serum albumin (BSA), polysulfone ( $\mathrm{Mw} \sim 60.000 \mathrm{~g} / \mathrm{mol}, \mathrm{Sp}^{2}$; Scientific Polymer Products, NY, USA), dimethylacetamide (DMAc, $99 \%$ ), hydrogen peroxide solution, L-cysteine, glutathione (Sigma Aldrich, St Louis, MO, USA), and sodium hydroxide pellet (Merck, NY, USA). Deionized water was used throughout the experiments. All reagents were used as received without further purification.

\section{Electrospinning of polysulfone nanofibers}

The polymer solution for the electrospinning process was prepared by dissolving polysulfone in DMAc $(35 \%, \mathrm{w} / \mathrm{v})$ at $60{ }^{\circ} \mathrm{C}$, followed by continuous stirring for $2 \mathrm{~h}$, to get a homogenous solution. Then, the prepared solution was taken in a $3 \mathrm{~mL}$ plastic syringe with a metallic needle (inner diameter: $0.8 \mathrm{~mm}$ ) and fixed horizontally in a syringe pump. This was followed by clamping the electrode of high-voltage power supply (SL series; Spellman, NY, USA) to the metal needle tip, where the collector was grounded. The flow rate of the polymer solution was optimized and controlled as $0.75 \mathrm{~mL} / \mathrm{h}$. The metal plate covered with an aluminum foil was used as a collector and the distance between the collector and the needle was optimized at $15 \mathrm{~cm}$. This setup was enclosed in a Plexiglas box, and electrospinning was carried out at $16 \mathrm{kV}, 22{ }^{\circ} \mathrm{C}$, and $20 \%$ relative humidity. The resulting nanofibers were collected and dried at room temperature under a fume hood overnight. Then, they were carefully separated from the aluminum foil for further process. The fibers were collected for the time period of $3 \mathrm{~h}$ and the thickness of the mat was $\sim 0.1 \mathrm{~mm}$. A similar procedure was carried out for collecting the nanofibers on a glass substrate for confocal laser scanning microscope (CLSM, Jena, Germany) measurement. This was done by placing the glass substrate on an aluminum foil covered metal plate.

\section{Preparation of fluorescent gold nanoclusters (AuNC)}

The AuNC were prepared according to the previously reported method with slight modification [29]. In brief, $10 \mathrm{mM}$ of $\mathrm{HAuCl}_{4}$ solution $(10 \mathrm{~mL})$ was mixed with an equal amount of BSA solution $\left(35 \mathrm{mg} \mathrm{mL}^{-1}\right)$ at $37^{\circ} \mathrm{C}$ under vigorous stirring. 
Two min later, $1 \mathrm{~mL} \mathrm{NaOH}(1 \mathrm{M})$ solution was introduced into the mixture, with further reaction under vigorous stirring at $37{ }^{\circ} \mathrm{C}$ for $12 \mathrm{~h}$. Under exposure to UV light $\left(\lambda_{\text {ext }}-366 \mathrm{~nm}\right)$, the prepared AuNC emitted red color, which was further decorated on the polysulfone nanofiber surface.

\section{Preparation of AuNC decorated nanofibrous membrane (FNFM)}

Dip coating technique was used for the decoration of AuNC on polysulfone nanofibrous membrane. The prepared electrospun nanofibrous membrane was immersed in a AuNC solution for $12 \mathrm{~h}$. Then, the membrane was taken from the solution carefully without damaging/ touching the surface and dried in air at room temperature. Further, adsorbed excess ligand from the surface of nanofibers was carefully removed by washing them with water for $2 \mathrm{~min}$ and then dried at room temperature. The prepared fluorescent nanofibrous membrane sample was called FNFM.

\section{Characterizations}

The morphology and diameter of the nanofibers at various stages were measured with a scanning electron microscope (SEM, Oregon, USA), Quanta 200 FEG and transmission electron microscopy (TEM) Tecnai G2 F30 (Oregon, USA). High angle, annular, dark field-scanning transmission electron microscopy (HAADF-STEM) elemental mapping was used to study the distribution of nanoclusters on the surface of nanofibers. The chemical composition and oxidation state of the elements in FNFM were studied by using X-ray photoelectron spectroscopy (XPS, West Sussex, UK) Thermo K-alphamonochromatic. Fluorescence emission spectra were measured by time-resolved fluorescence spectrophotometer (FL1057 TCSPC, NJ, USA). The fluorescence imaging of FNFM were captured by using confocal laser scanning microscopy (CLSM, Jena, Germany) Zeiss LSM 510, $\lambda_{\text {ext }}-488 \mathrm{~nm}, 20 \times$ magnification. The absorption spectra were recorded before and after treatment with $\mathrm{H}_{2} \mathrm{O}_{2}$ using UV-Vis spectroscopy (Varian, Cary 100, Middelburg, The Netherlands). Circular dichroism system (JASCO, J-815, Tokyo, Japan) was used to record the $\mathrm{CD}$ spectra.

\section{Visual colorimetric detection of $\mathrm{H}_{2} \mathrm{O}_{2}$}

A typical colorimetric analysis for $\mathrm{H}_{2} \mathrm{O}_{2}$ was performed as follows. Different concentrations of $\mathrm{H}_{2} \mathrm{O}_{2}$ were prepared by diluting the stock solution. First, the $\mathrm{H}_{2} \mathrm{O}_{2}$ was added in AuNC solution to check the color change. Then the flexible FNFM was cut into small pieces, $2 \times 2 \mathrm{~cm}$, and dipped in different concentrations of $\mathrm{H}_{2} \mathrm{O}_{2}$ separately. The obvious color change from bright red to blue was noticed under UV light. A similar procedure was also carried out for glutathione (GSH), L-cysteine (Cys), sodium $\left(\mathrm{Na}^{+}\right)$, potassium $\left(\mathrm{K}^{+}\right)$, and calcium $\left(\mathrm{Ca}^{2+}\right)$ as interference. All the experiments were conducted in triplicate and the mean values were reported. For CLSM, the desired concentration of $\mathrm{H}_{2} \mathrm{O}_{2}$ solution $(2 \mu \mathrm{L})$ was dropped on the FNF and then images were taken after trying it. Also, the fluorescence intensity was measured across the FNF surface. The excitation source was fixed at $488 \mathrm{~nm}$ and the images were captured at $20 \times$ magnification.

Fig. 1 (a) HAADF-STEM (highangle annular dark-field scanning transmission electron microscopy) image of single FNF and their corresponding mapping images of the elements, (b) carbon, (c) oxygen, (d) sulfur, (e) nitrogen, and (f) gold

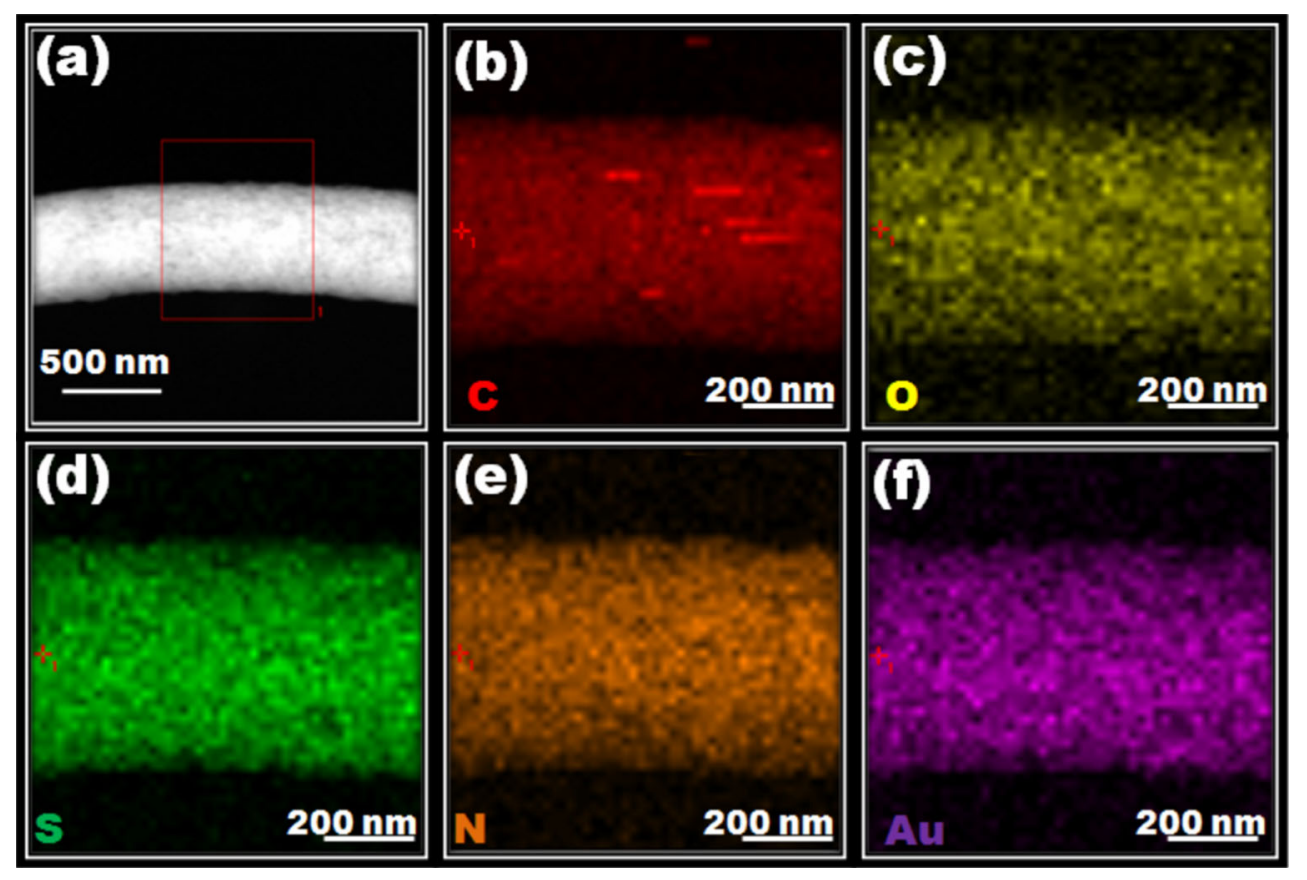




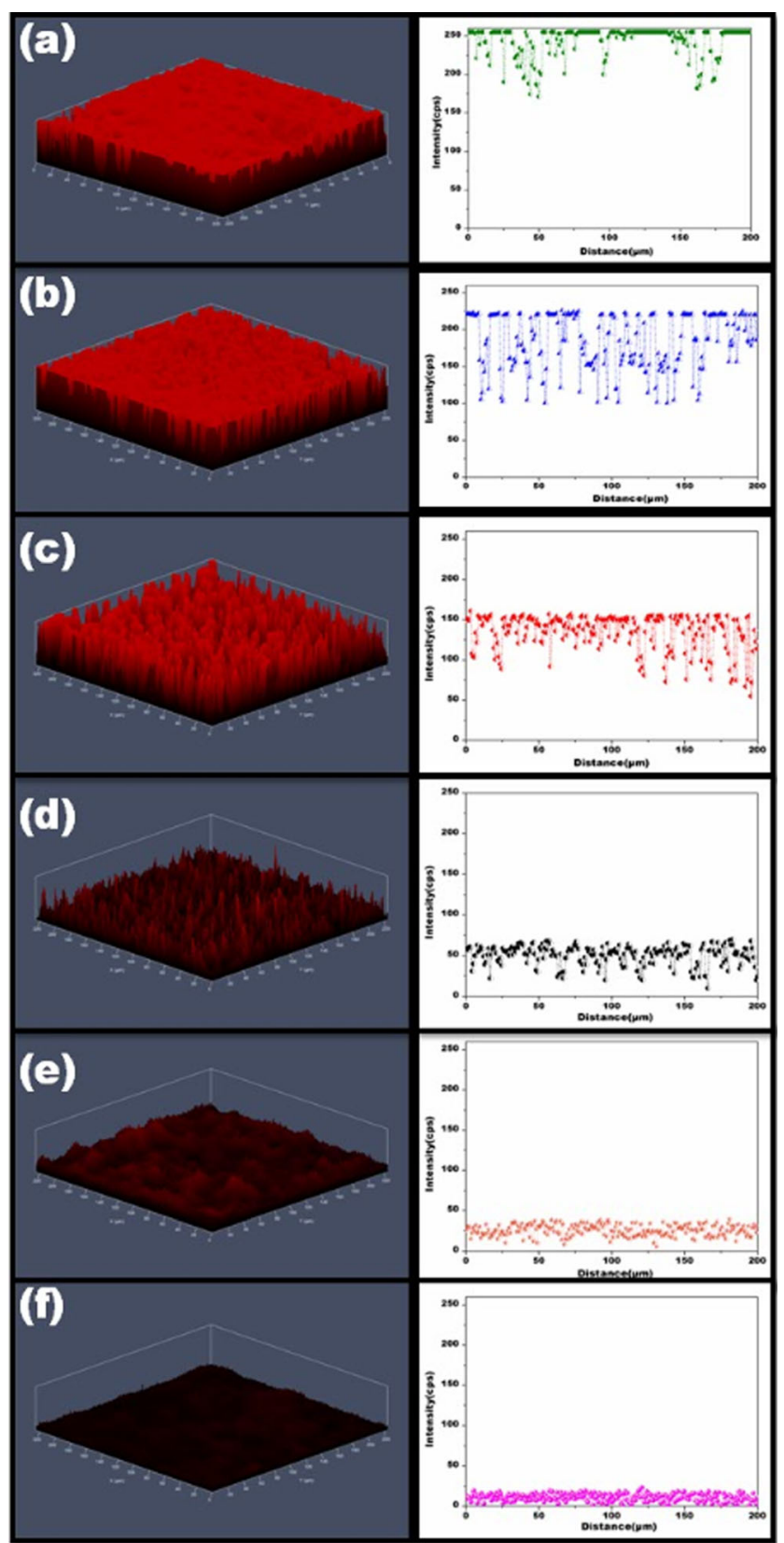

Fig. 2 Confocal laser scanning microscopy (CLSM) images of FNFM before (a) and after exposure of different concentrations of $\mathrm{H}_{2} \mathrm{O}_{2}$ and their intensity profiles collected across the fiber surface (b) $500 \mathrm{nM}$, (c) $1 \mu \mathrm{M}$, (d) $10 \mu \mathrm{M}$, (e) $50 \mu \mathrm{M}$, and (f) $100 \mu \mathrm{M}$

\section{Results and discussion}

Fabrication of FNFM sensor strip was performed by decorating $\mathrm{AuNC}$ on the electrospun nanofibrous membrane, allowing uniform distribution of AuNC on the nanofiber surface and further enabling maximal exposure to the target analytes. The size of the prepared AuNC was found to be $\sim 1.5$ to $3 \mathrm{~nm}$ and having lattice spacing of $0.24 \mathrm{~nm}$, corresponds to the $\mathrm{d}$ spacing of (111) orientation of $\mathrm{Au}$ as shown in Electronic Supplementary Material (ESM) Fig. S1, [30]. The circular dichroism (CD) spectra of AuNC exhibited two characteristic peaks of an $\alpha$-helical protein structure of BSA positioned at $\sim 206 \mathrm{~nm}$ and $221 \mathrm{~nm}$, which corresponds to $\pi-\pi^{*}$ and $\mathrm{n}-\pi^{*}$ transitions of the peptide bonds on the $\alpha$-helices, respectively (see ESM Fig. S2), [31]. As the distinct features of AuNC are known, we concentrated our focus on understanding their rapid sensing behavior and mechanism. ESM Fig. S3 details the morphology of the electrospun polysulfone nanofibers before and after decoration of AuNC (i.e., FNFM). It can be learned from the figure that the nanofibers are free of defect and randomly oriented with a relatively uniform diameter of $526 \pm 70 \mathrm{~nm}$ and $648 \pm 236 \mathrm{~nm}$, respectively. The fibrous morphology of the sample has not been changes after decoration of AuNC; however, the smooth nature of the fiber surface was slightly affected and fiber diameter was increased slight possibly due to the swelling of the fibers. Further, scanning transmission electron microscopy (STEM) elemental mapping of single FNFM confirmed the uniform distribution of AuNC on nanofiber surface without any agglomeration as depicted in Fig. 1. Interestingly, no adsorption of excess protein was noticed. The prepared FNFM showed intense red color under exposure to UV light $\left(\lambda_{\text {ext }}-366 \mathrm{~nm}\right)$ as shown in ESM Fig. S4. It has been found that the uniform distribution of AuNC improves the homogeneity of the color, which is a principal characteristic in colorimetric sensing. Also, the FNFM is highly flexible in nature, even after decoration AuNC as show in ESM Fig. S4. This can be effectively put to use in sensing applications similar to $\mathrm{pH}$ paper.

A vital attribute of any colorimetric indicator in practical application is its ease of handling. However, most colorimetric-based sensors in previous reports were not suitable for use in biological and environmental-friendly applications because the technology to date has relied upon fluorescent probes suspended in solution. Interestingly, this can be overcome by assembling them on a support matrix. This is likely to include the following advantages: (i) reducing the amount of probe, which is expected to reduce the cost of the sensor and lower detection limit; (ii) increasing the life time. The stability of the fluorescent probe is limited in solution phase as suspended probes have tendency to aggregate (iii) In addition, the effect of environmental factors on the properties and fluorescence characteristics of these probes.

Rapid colorimetric sensing proves to be challenging in the way that macroscopic color changes are seldom coupled to the rate of interaction and their properties of a system. Solution-based assays are prone to containment issues such as spillage and disposal after use as a sensor for detecting pollutants. In order to improve the usage and sensing performance of fluorescent probes, its availability should be increased, and more stability is encouraged. Combining all these facts, we have devised our proposed method highlighting several 


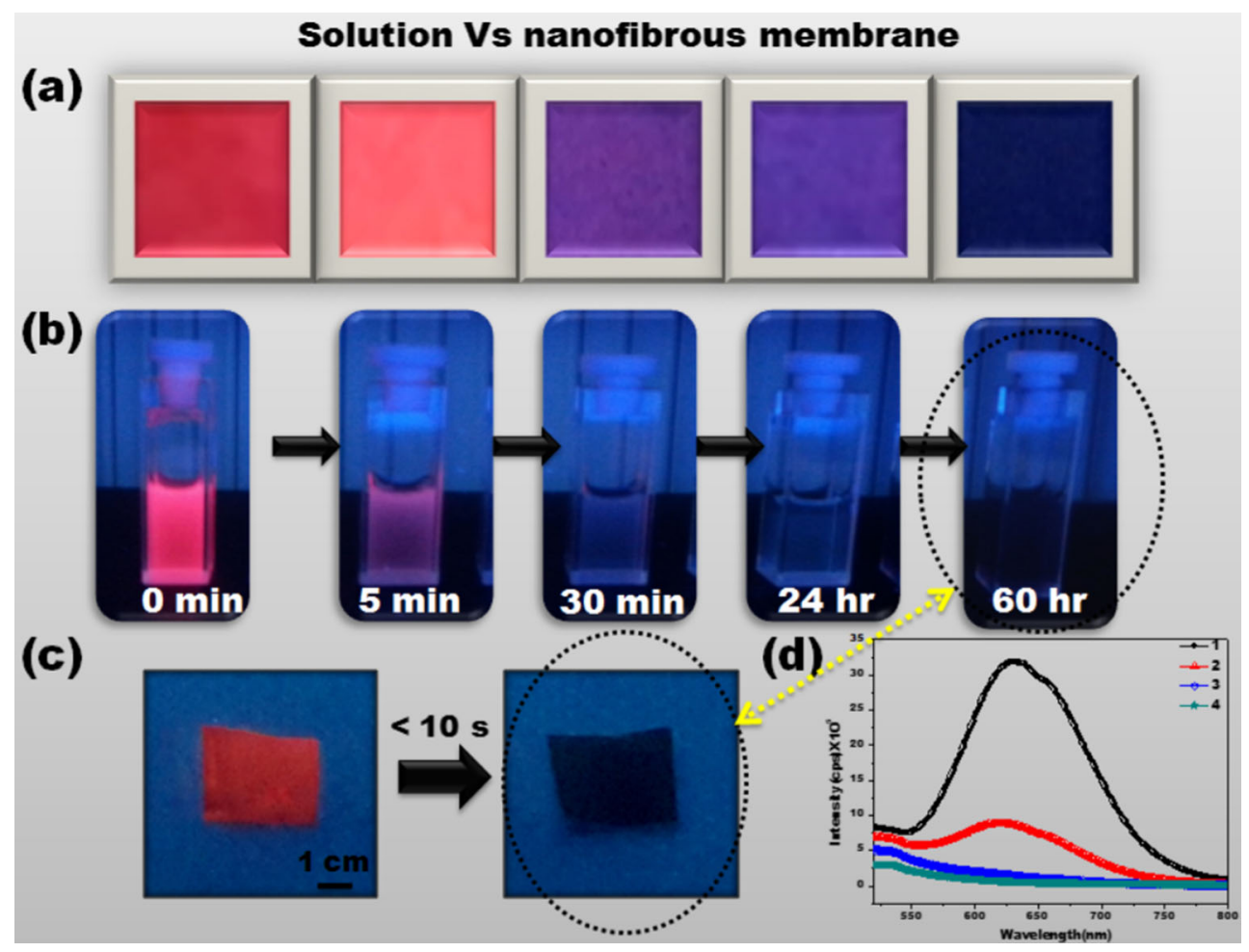

Fig. 3 Visual colorimetric sensing performance of FNFM and their comparative study with AuNC solution, (a) optical colorimetric response of FNFM upon treatment with $50 \mu \mathrm{M}, 100 \mu \mathrm{M}, 1 \mathrm{mM}$, and $10 \mathrm{mM} \mathrm{H}_{2} \mathrm{O}_{2}$ from left to right, respectively. The color of the FNFM gradually changed to blue upon increasing the concentration of $\mathrm{H}_{2} \mathrm{O}_{2}$; (b) Time-dependent sensing performance of AuNC solution in the

key advantages: it is simple and fast as it involves the decoration of AuNC on the nanofiber surface, contributing a greater chance to interact with $\mathrm{H}_{2} \mathrm{O}_{2}$. This provides the possibility of observing the clear color change presence of $100 \mathrm{mM} \mathrm{H}_{2} \mathrm{O}_{2}$; photographs were taken at different time periods under UV light $\left(\left(\lambda_{\text {ext }}=366 \mathrm{~nm}\right)\right.$; (c) $100 \mathrm{mM} \mathrm{H}_{2} \mathrm{O}_{2}$ treated FNFM, color change has occurred within $10 \mathrm{~s}$. The observed quick color change in the FNFM is comparable to the finding at $60 \mathrm{~h}$ in solution phase AuNC. (d) The normalized PL emission spectra of FNFM (1) and $\mathrm{H}_{2} \mathrm{O}_{2}$ treated FNFM with different concentration $50 \mu \mathrm{M}$ (2), $100 \mu \mathrm{M}$ (3), and $1 \mathrm{mM}$ (4)
Fig. 4 Selective sensing response of FNFM after incubation for $30 \mathrm{~min}$ in the presence of various biomolecules. The variation in the relative fluorescence intensity $\left(\mathrm{I}_{0} / \mathrm{I}\right.$, where I and $\mathrm{I}_{0}$ are the fluorescence intensity in the presence and absence of molecules, respectively) of FNFM at a preset concentration of $10 \mathrm{mM}$. Inset: photographs showing the selectivity of FNFM taken under UV light exposure. The error bar indicates standard deviation of three replicates

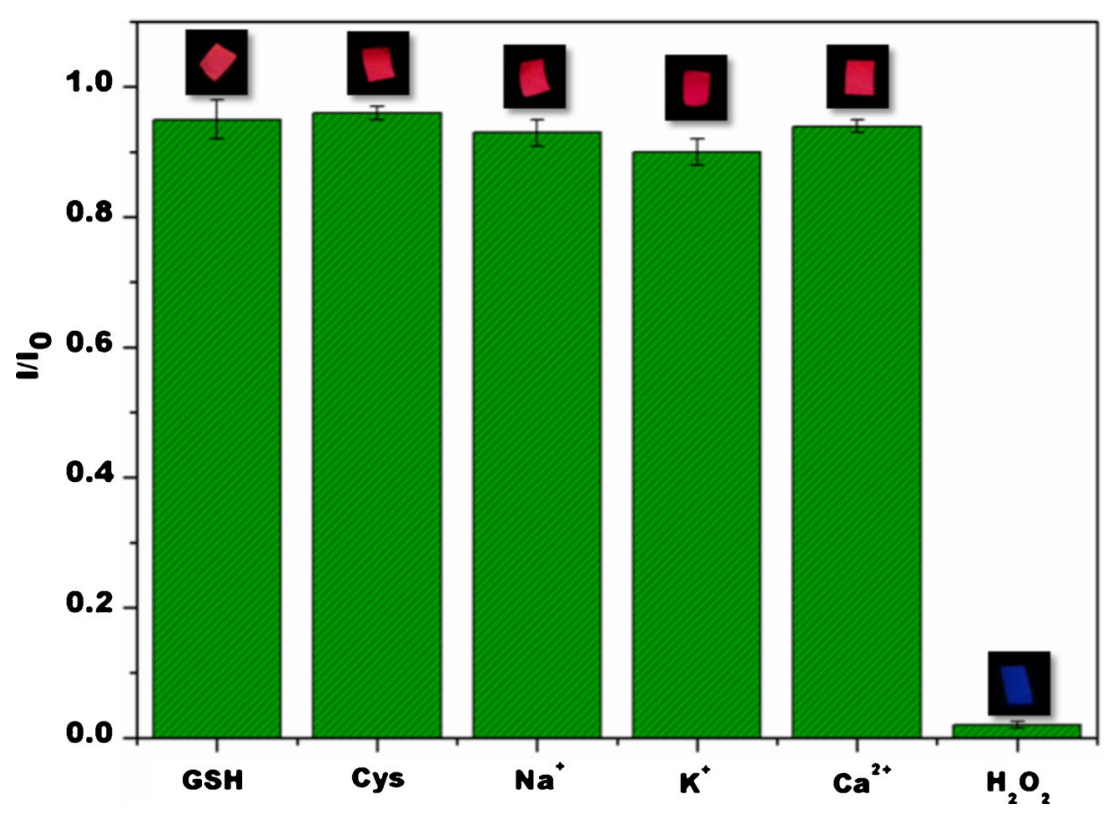


The visual colorimetric response of the fluorescence nanofibers towards $\mathrm{H}_{2} \mathrm{O}_{2}$ is studied using contact-mode and CLSM based analysis. The authentic red emission characteristics of AuNC are clearly viewed under CLSM. The noticed fluctuation in intensity profile is due to the collection of data across the randomly oriented fibrous structure on glass substrate. As seen in Fig. 2, it can be observed that the red fluorescence and its intensity profile of FNF were gradually decreased upon increasing the concentration of $\mathrm{H}_{2} \mathrm{O}_{2}$. The CLSM-based analysis enabled the sensing of $\mathrm{H}_{2} \mathrm{O}_{2}$ with a detection limit of 500 $\mathrm{nM}$. The observed detection limit is comparable to previous reports [18, 32, 33]. Further, contact mode approach has been adopted to study the visual colorimetric response of the flexible FNFM towards various concentrations of $\mathrm{H}_{2} \mathrm{O}_{2}$. This is achieved by cutting the FNFM into small pieces and dipping it in various concentrations of $\mathrm{H}_{2} \mathrm{O}_{2}$ separately for about $1 \mathrm{~min}$. Subsequently, a distinct color change from red to purple, followed by a bluish violet has been observed, detectable with the naked eye up to $1 \mathrm{mM}$, highlighting time- and dosedependent responses as clearly indicated in Fig. 3a.

It would be highly appreciated if we can effectively reproduce the sensor strip for several attempts without failing their sensing response. The FNFM fabricated from different batches exhibit similar sensing responses, implying the consistency of its performance. Encouraged by seeing an enormous number of annotations on AuNC in colorimetric sensor, we are highly motivated to investigate and compare the sensing performance of FNFM with AuNC in solution phase. It is anticipated that the sensing performance of fluorescent probes in solution phase could be higher than the solid matrix platform. In order to confirm this prediction, the AuNC in solution phase and decorated on nanofibrous membrane (i.e., FNFM were exposed to $\mathrm{H}_{2} \mathrm{O}_{2}$ at a concentration of $100 \mathrm{mM}$ ). Initially, prepared AuNC was diluted one-fold $(0.5 \mathrm{~mL}+0.5 \mathrm{~mL})$ followed by the addition of an equal volume of $\mathrm{H}_{2} \mathrm{O}_{2}$ solution $(1 \mathrm{~mL})$ to study the sensing performance at solution phase.

The change in visual color with definite periods of incubation time $(0,5,30 \mathrm{~min}$, and 24 and $60 \mathrm{~h})$ was monitored under UV light $\left(\lambda_{\text {ext }}=366 \mathrm{~nm}\right)$ and normal light condition, and photographs were taken as shown in Fig. 3b. Interestingly, the quenching fluorescence nature of AuNC in aqueous phase was time-dependent. The observation at $5 \mathrm{~min}$ showed a slight change in fluorescence and at the end of $60 \mathrm{~h}$, the color of the solution changed to deep purple. Conversely, an ultrafast quenching of fluorescence was observed in the FNFM less than $10 \mathrm{~s}$ following exposure to same concentration of $\mathrm{H}_{2} \mathrm{O}_{2}$ as illustrated in Fig. 3c. The observed color change in the FNFM is comparable to the finding at $60 \mathrm{~h}$ in solution phase.

To demonstrate the enhanced rapid sensing performance of FNFM compared with solution phase, we captured a real time video during $\mathrm{H}_{2} \mathrm{O}_{2}$ exposure as demonstrated in Video $\mathrm{S}$. The color change occurred immediately after the membrane comes in contact with $\mathrm{H}_{2} \mathrm{O}_{2}$ solution. It is obvious that the sensing performance of FNFM is much higher at a shorter exposure time than in the solution state. The observed ultrafast quenching response is attributable to the enhanced interaction between the fluorescent probe located on the surface of nanofibers and $\mathrm{H}_{2} \mathrm{O}_{2}$. The larger surface area of the nanofibers serves as an ideal platform for AuNC resulting in increased interaction with analytes. This also facilitates the easy removal of free and excess protein on the AuNC and FNFM surface without affecting their characteristics. In solution state, the surplus amount of free and excess protein is expected to

Fig. 5 (a) Transmission electron microscopy (TEM) image of $100 \mathrm{mM} \mathrm{H}_{2} \mathrm{O}_{2}$ treated single FNF; (b) UV-Vis absorption spectra of AuNC before and after treatment with $100 \mathrm{mM} \mathrm{H}_{2} \mathrm{O}_{2}$. Inset: visual color change of AuNC in the presence of $\mathrm{H}_{2} \mathrm{O}_{2}$. The photographs were taken under normal light condition. (c) HAADF-STEM image of $100 \mathrm{mM}$ treated single FNF and their corresponding mapping images of elements
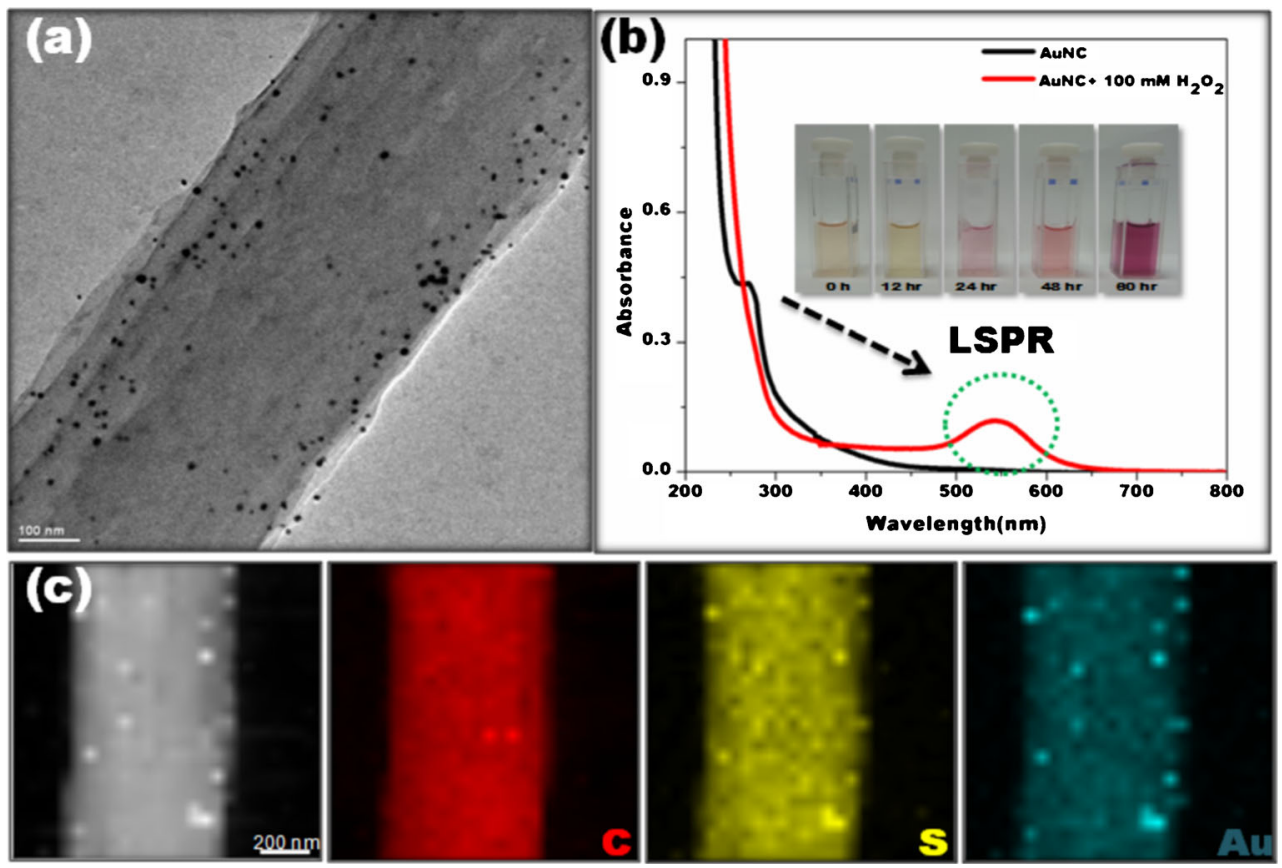
reduce/slow the interaction with $\mathrm{H}_{2} \mathrm{O}_{2}$. As shown in Fig. 3d, the change in fluorescence characteristics of FNFM upon treatment with various concentrations of $\mathrm{H}_{2} \mathrm{O}_{2}$ has been clearly noticed. By comparing the spectra, a blue shift has been noticed from 640 to $630 \mathrm{~nm}$, which might have resulted from the ligand-induced aggregation of gold nanoclusters $[34,35]$.

The specificity and selectivity of FNFM towards $\mathrm{H}_{2} \mathrm{O}_{2}$ was examined over common biological molecules, such as glutathione (GSH), cysteine (Cys), $\mathrm{Na}^{+}, \mathrm{K}^{+}$, and $\mathrm{Ca}^{2+}$ at a preset concentration $(10 \mathrm{mM})$. In brief, FNFM was immersed separately in analyte solution for about $10 \mathrm{~min}$ and air-dried. The visual color change was observed under UV lamp and photographs were taken. The variation in the relative fluorescence intensity $\left(\mathrm{I}_{0} / \mathrm{I}\right.$, where $\mathrm{I}$ and $\mathrm{I}_{0}$ are the fluorescence intensity in the presence and absence of molecules, respectively) of FNFM is shown in Fig. 4. The FNFM exhibited dark blue color under exposure to $\mathrm{H}_{2} \mathrm{O}_{2}$, whereas no obvious significant change in the color was noted for other common biomolecules as illustrated in Fig. 4, inset. Subsequently, we studied the possible mechanism for rapid colorimetric sensing of $\mathrm{H}_{2} \mathrm{O}_{2}$. The combination in the variations of the TEM images and UV spectra compared with native FNFM revealed the transition of gold nanoclusters to nanoparticles, under exposure to $\mathrm{H}_{2} \mathrm{O}_{2}$ as shown in Fig. 5a and b. The TEM image and elemental mapping of $100 \mathrm{mM} \mathrm{H}_{2} \mathrm{O}_{2}$ - treated single FNF is shown in Fig. 5c. This observed result reveals the existence of most of the gold nanoparticles as single with extremely low degree of aggregation. It is interesting to note that the nanofiber structure did not degrade, which is further confirmed by SEM image as shown in ESM Fig. S5. Also, the flexibility of FNFM is well maintained as shown in ESM Fig. S6.

The UV-Vis absorption spectra of AuNC before and after treatment with $\mathrm{H}_{2} \mathrm{O}_{2}$ are shown in Fig. 5b. Compared with two spectra, it is clearly seen that the LSPR peak is not observed in AuNC, which is closely related to the properties of nanoclusters [36, 37]. The appearance of LSPR bands is directly correlated with the formation of gold nanoparticles and not through an aggregation process. Upon addition of $100 \mathrm{mM} \mathrm{H}_{2} \mathrm{O}_{2}$, the red fluorescence of AuNC solution decreases gradually, and emitted light to dark blue color depends on the incubation time period. Under daylight condition, the AuNC solution gradually changes its color from transparent to light red and then dark wine. The observed distinct color difference is shown as an inset in Fig. $5 b$.

A rapid deterioration of BSA structure and its characteristics fluorescence might be expected when exposed to $\mathrm{H}_{2} \mathrm{O}_{2}$ since thiol group present in BSA plays a significant role in stabilizing the AuNC [38]. Hence, to substantiate this, efforts were made to study the effect of $\mathrm{H}_{2} \mathrm{O}_{2}$ on fluorescence characteristics of BSA in FNFM. As shown in ESM Fig. S7, a gradual decrease in the fluorescence intensity was observed, and upon increasing the concentration, the intensity was completely quenched. Also, it has been found that the $\mathrm{H}_{2} \mathrm{O}_{2}$

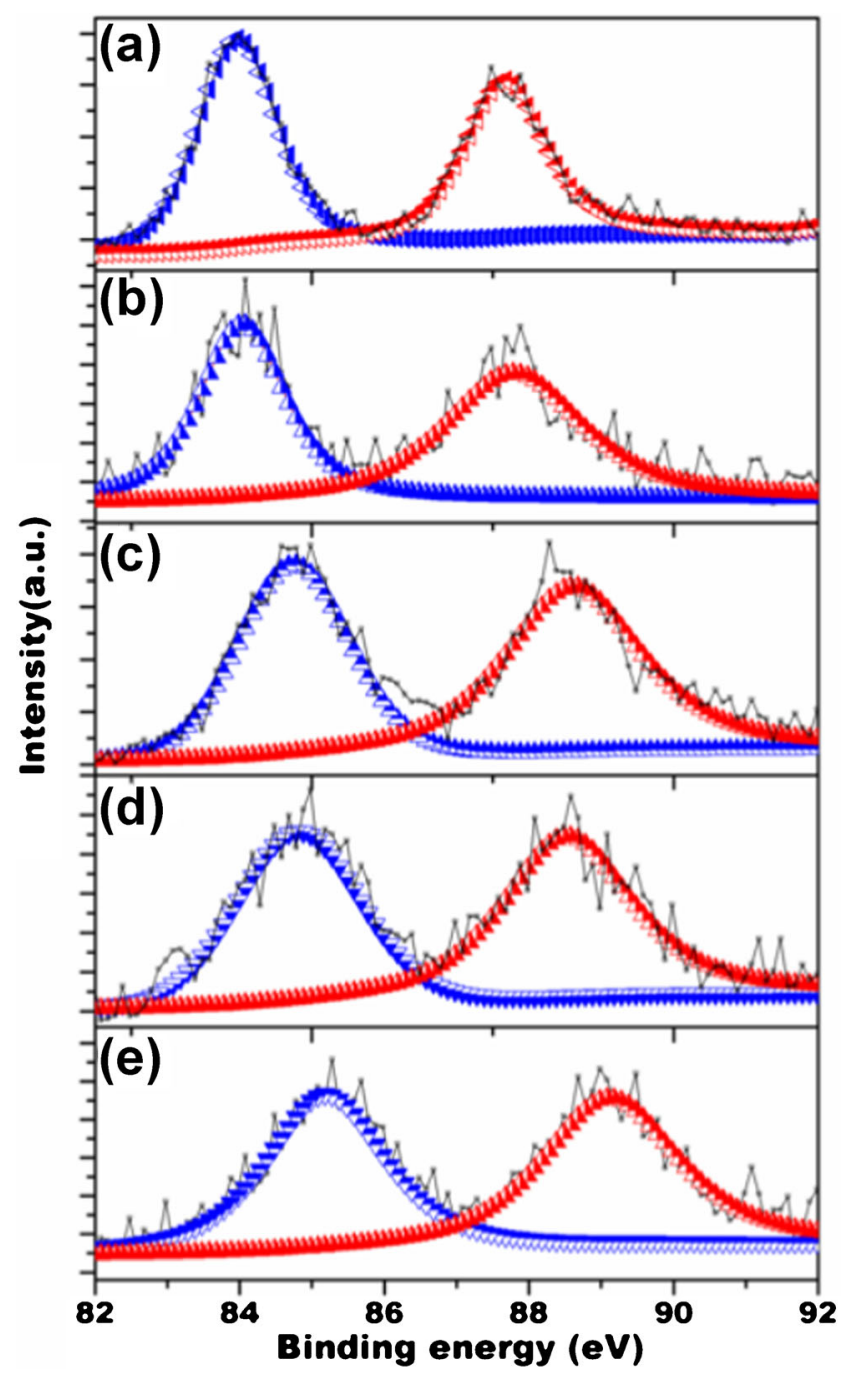

Fig. 6 Deconvoluted $\mathrm{Au}(4 \mathrm{f})$ spectra of FNFM before (a) and after addition of $\mathrm{H}_{2} \mathrm{O}_{2}$ with different concentration, (b) $100 \mu \mathrm{M}$, (c) $1 \mathrm{mM}$, (d) $10 \mathrm{mM}$, and (e) $100 \mathrm{mM}$

not only affects the $\mathrm{Au}-\mathrm{S}$ bonding in the gold nanoclusters but also severely affects the free BSA. Further, the oxidation state of the $\mathrm{Au}$ in FNFM was studied before and after the addition of $\mathrm{H}_{2} \mathrm{O}_{2}$ at different concentrations. As depicted in Fig. 6, the XPS spectrum in the absence of $\mathrm{H}_{2} \mathrm{O}_{2}$ shows an $\mathrm{Au} 4 \mathrm{f}$ band at binding energies of 83.9 and $87.6 \mathrm{eV}$ assigned to $4 \mathrm{f}_{5 / 2}$ and $4 f_{7 / 2}$, which could be attributed to $\mathrm{Au}(0)$ state [39]. When reacted with $\mathrm{H}_{2} \mathrm{O}_{2}$, the peak gradually shifts to a higher binding energy level, which can be ascribed to higher oxidation states of $\mathrm{Au}$ [33]. A similar finding was also observed in AuNC solution when reacted with $\mathrm{H}_{2} \mathrm{O}_{2}$ as depicted in ESM Fig. S8.

\section{Conclusion}

We have developed an efficient, sensitive, easy to-use, and flexible FNFM for rapid detection of $\mathrm{H}_{2} \mathrm{O}_{2}$. To the best of 
our knowledge, rapid visual colorimetric detection of $\mathrm{H}_{2} \mathrm{O}_{2}$ by using flexible electrospun membrane has been reported for the first time. The FNFM was successfully fabricated by decorating the gold nanocluster on the surface of electrospun polysulfone nanofibers. The sensing performance was studied by using CLSM and contact mode colorimetric approaches. The limit of detection (LOD) was determined to be $500 \mathrm{nM}$. The observed visual colorimetric results show that increasing the $\mathrm{H}_{2} \mathrm{O}_{2}$ concentration leads to change in the color from red to blue, and subsequently decreasing their fluorescence intensity by releasing ligands from the AuNC surface. A quick color change appears after FNFM comes in contact with $\mathrm{H}_{2} \mathrm{O}_{2}$, which is strongly supported by video. The sensing performance was systematically compared with AuNC solution and the findings prove the advantage of nanofibrous membrane as a matrix to enhance sensing performance and facilitate rapid detection. Studies suggest that the sensing mechanism is based on the interaction of $\mathrm{H}_{2} \mathrm{O}_{2}$ with BSA molecules than with gold. The changes observed in the UV absorption spectra were studied, highlighting the disappearance of the absorption peak in the gold nanoclusters and the emergence of a new peak, called the LSPR band. The change in the morphology was studied by using TEM and STEM-EDX mapping. The flexibility, along with the visual color change of FNFM assured the practical applicability. The reported sensor system also proves the technical feasibility and provides enhanced colorimetric sensing using nanofibrous structure, thereby offering plenty of room to improve interaction with $\mathrm{H}_{2} \mathrm{O}_{2}$.

Acknowledgments S.A. and B.B. thank the Scientific and Technological Research Council of Turkey (TÜBITAK) (TÜBITAK-BIDEB 2216, Research Fellowship Programme for Foreign Citizens) for postdoctoral fellowship funding. Z.A. thanks to TUBITAK-BIDEB (2211-C) for national PhD scholarship and TUBITAK (project no. 213 M185) for PhD scholarship. T.U acknowledges funding support of FP7-Marie Curie International Reintegration Grant (IRG) for funding NANOWEB (PIRG06GA-2009-256428) and partial support of The Turkish Academy of Sciences - Outstanding Young Scientists Award Program (TUBA-GEBIP). The authors thank M. Guler for TEM-STEM technical support.

\section{Compliance with ethical standards}

Conflict of interest The authors declare that they have no conflict of interest.

\section{References}

1. Chen W, Cai S, Ren QQ, Wen W, Zhao YD (2012) Recent advances in electrochemical sensing for hydrogen peroxide: a review. Analyst 137:49-58
2. Chen X, Wu G, Cai Z, Oyama M, Chen X (2014) Advances in enzyme-free electrochemical sensors for hydrogen peroxide, glucose, and uric acid. Microchim Acta 181:689-705

3. Zhang P, Wang H, Zhang X, Xu W, Li Y, Li Q, Wei G, Su Z (2015) Graphene film doped with silver nanoparticles: self-assembly formation, structural characterizations, antibacterial ability, and biocompatibility. Biomater Sci 3:852-860

4. Dickinson BC, Chang CJ (2008) A targetable fluorescent probe for imaging hydrogen peroxide in the mitochondria of living cells. J Am Chem Soc 130:9638-9639

5. Chen Y, Shen Y, Sun D, Zhang H, Tian D, Zhang J, Zhu JJ (2011) Fabrication of a dispersible graphene/gold nanoclusters hybrid and its potential application in electrogenerated chemiluminescence. Chem Commun 47:11733-11735

6. Zhao Q, Chen S, Huang H, Zhang L, Wang L, Liu F, Chen J, Zeng Y, Chu PK (2014) Colorimetric and ultra-sensitive fluorescence resonance energy transfer determination of $\mathrm{H}_{2} \mathrm{O}_{2}$ and glucose by multi-functional Au nanoclusters. Analyst 139:1498-1503

7. Zhang J, Tu L, Zhao S, Liu G, Wang Y, Wang Y, Yue Z (2015) Fluorescent gold nanoclusters based photoelectrochemical sensors for detection of $\mathrm{H}_{2} \mathrm{O}_{2}$ and glucose. Biosens Bioelectron 67: 296-302

8. Li J, Guo LR, Gao W, Xia XH, Zheng LM (2009) Enhanced electrochemiluminescence efficiency of Ru(II) derivative covalently linked carbon nanotubes hybrid. Chem Commun 7545-7547

9. Xu M, Han JM, Wang C, Yang X, Pei J, Zang L (2014) Fluorescence ratiometric sensor for trace vapor detection of hydrogen peroxide. ACS Appl Mater Interfaces 6:8708-8714

10. Wen F, Dong Y, Feng L, Wang S, Zhang S, Zhang X (2011) Horseradish peroxidase functionalized fluorescent gold nanoclusters for hydrogen peroxide sensing. Anal Chem 83: $1193-1196$

11. You X, Li Y (2015) Direct chemiluminescence of fluorescent gold nanoclusters with classic oxidants for hydrogen peroxide sensing. Arab J Chem. doi:10.1016/j.arabjc.2015.05.019

12. Li J, Mei H, Zheng W, Pan P, Sun XJ, Li F, Guo F, Zhou HM, Ma JY, Xu XX, Zheng YF (2014) A novel hydrogen peroxide biosensor based on hemoglobin-collagen-CNTs composite nanofibers. Colloids Surf B: Biointerfaces 118:77-82

13. Wu Y, Huang J, Zhou T, Rong M, Jiang Y, Chen X (2013) A novel solid-state electrochemiluminescence sensor for the determination of hydrogen peroxide based on an Au nanocluster-silica nanoparticle nanocomposite. Analyst 138:5563-5565

14. Zhang Y, Yang W, Wang Y, Jia J, Wang J (2013) Nonenzymatic hydrogen peroxide sensor based on a glassy carbon electrode modified with electrospun $\mathrm{PdO}-\mathrm{NiO}$ composite nanofibers. Microchim Acta 180:1085-1091

15. Anitha S, Brabu B, Rajesh KP, Natarajan TS (2013) Fabrication of UV sensor based on electrospun composite fibers. Mater Lett 92: $417-420$

16. Xiao X, Song Y, Liu H, Xie M, Hou H, Wang L, Li Z (2013) Electrospun carbon nanofibers with manganese dioxide nanoparticles for nonenzymatic hydrogen peroxide sensing. J Mater Sci 48: $4843-4850$

17. Xu D, Li L, Ding Y, Cui S (2015) Electrochemical hydrogen peroxide sensors based on electrospun $\mathrm{La}_{0.7} \mathrm{Sr}_{0.3} \mathrm{Mn}_{0.75} \mathrm{Co}_{0.25} \mathrm{O}_{3}$ nanofiber modified electrodes. Anal Methods 7:6083-6088

18. Lei Z, Liu X, Ma L, Liu D, Zhang H, Wang Z (2015) Spheres-onsphere silica microspheres as matrix for horseradish peroxidase immobilization and detection of hydrogen peroxide. RSC Adv 5: 38665-38672

19. Khalid W, El HM, Murböck T, Yue Z, Montenegro JM, Schubert K, Göbel G, Lisdat F, Witte G, Parak WJ (2011) Immobilization of quantum dots via conjugated self-assembled monolayers and their application as a light-controlled sensor for the detection of hydrogen peroxide. ACS Nano 5:9870-9876 
20. Liu Q, Zhang T, Yu L, Jia N, Yang DP (2013) 3D nanoporous Ag@BSA composite microspheres as hydrogen peroxide sensors. Analyst 138:5559-5562

21. Deng M, Xu S, Chen F (2014) Enhanced chemiluminescence of the luminol-hydrogen peroxide system by BSA-stabilized $\mathrm{Au}$ nanoclusters as a peroxidase mimic and its application. Anal Methods 6:3117-3123

22. Senthamizhan A, Celebioglu A, Uyar T (2015) Real-time selective visual monitoring of $\mathrm{Hg}^{2+}$ detection at ppt level: an approach to lighting electrospun nanofibers using gold nanoclusters. Sci Rep 5:10403

23. Senthamizhan A, Uyar T (2015) In: Macagnano A, Zampetti E, Kny E (eds) Electrospinning for High Performance Sensors, 1st edn. Switzerland, Springer International Publishing

24. Senthamizhan A, Celebioglu A, Uyar T (2015) Ultrafast on-site selective visual detection of TNT at sub-ppt level using fluorescent gold cluster incorporated single nanofiber. Chem Commun 51: $5590-5593$

25. Senthamizhan A, Celebioglu A, Uyar T (2014) Flexible and highly stable electrospun nanofibrous membrane incorporating gold nanoclusters as an efficient probe for visual colorimetric detection of $\mathrm{Hg}(\mathrm{II})$. J Mater Chem A 2:12717-12723

26. Su Z, Dinga J, Wei G (2014) Electrospinning: a facile technique for fabricating polymeric nanofibers doped with carbon nanotubes and metallic nanoparticles for sensor applications. RSC Adv 4:5259852610

27. Andrade PF, de Faria AF, Oliveira SR, Arruda MA, Gonçalves Mdo C (2015) Improved antibacterial activity of nanofiltration polysulfone membranes modified with silver nanoparticles. Water Res 81:333-342

28. Dahe GJ, Kadam SS, Sabale SS, Kadam DP, Sarkate LB, Bellare JR (2011) In vivo evaluation of the biocompatibility of surface modified hemodialysis polysulfone hollow fibers in rat. PLoS One 6, e25236

29. Xie J, Zheng Y, Ying JY (2009) Protein-directed synthesis of highly fluorescent gold nanoclusters. J Am Chem Soc 131:888-889
30. Yan L, Cai Y, Zheng B, Yuan H, Guo Y, Xiao D, Choi MMF (2012) Microwave-assisted synthesis of BSA-stabilized and HSAprotected gold nanoclusters with red emission. J Mater Chem 22: $1000-1005$

31. Zheng C, Wang H, Xu W, Xu C, Liang J, Han H (2014) Study on the interaction between histidine-capped Au nanoclusters and bovine serum albumin with spectroscopic techniques. Spectrochim Acta A Mol Biomol Spectrosc 118:897-902

32. Wen T, Qu F, Li NB, Luo HQ (2012) Polyethyleneimine-capped silver nanoclusters as a fluorescence probe for sensitive detection of hydrogen peroxide and glucose. Anal Chim Acta 749:56-62

33. Molaabasi F, Hosseinkhani S, Moosavi-Movahedi AA, Shamsipur M (2015) Hydrogen peroxide sensitive hemoglobin-capped gold nanoclusters as a fluorescence enhancing sensor for the label-free detection of glucose. RSC Adv 5:33123-33135

34. Li G, Zhu D, Liu Q, Xue L, Jiang H (2013) Rapid detection of hydrogen peroxide based on aggregation induced ratiometric fluorescence change. Org Lett 15:924-927

35. Wang F, Liu X, Lu CH, Willner I (2013) Cysteine-mediated aggregation of $\mathrm{Au}$ nanoparticles: the development of a $\mathrm{H}_{2} \mathrm{O}_{2}$ sensor and oxidase-based biosensors. ACS Nano 7:7278-7286

36. Raut S, Chib R, Rich R, Shumilov D, Gryczynski Z, Gryczynski I (2013) Polarization properties of fluorescent BSA protected $\mathrm{Au}_{25}$ nanoclusters. Nanoscale 5:3441-3446

37. Zhou Z, Zhang C, Qian Q, Ma J, Huang P, Zhang X, Pan L, Gao G, Fu H, Fu S, Song H, Zhi X, Ni J, Cui D (2013) Folic acidconjugated silica capped gold nanoclusters for targeted fluorescence/X-ray computed tomography imaging. J Nanobiotechnol 11:17

38. Jin L, Shang L, Guo S, Fang Y, Wen D, Wang L, Yin J, Dong S (2011) Biomolecule-stabilized Au nanoclusters as a fluorescence probe for sensitive detection of glucose. Biosens Bioelectron 26: 1965-1969

39. Le Guével X, Hötzer B, Jung G, Hollemeyer K, Trouillet V, Schneider M (2011) Formation of fluorescent metal (Au, Ag) nanoclusters capped in bovine serum albumin followed by fluorescence and spectroscopy. J Phys Chem C 115:10955-10963 